\title{
Histopathological Evaluation of Scar Tissue and Adjacent Non-Involved Skin
} Rehab Monir Samaka ${ }^{1}$, Ola Ahmed Bakry ${ }^{2}$, Nancy Mohammed Fayez ${ }^{3}$, Iman Seleit ${ }^{2}$

${ }^{1}$ Department of Pathology, Faculty of Medicine, Menoufia University, Shebin Elkom, Menoufia Governorate ${ }^{2}$ Department of Dermatology, Andrology and STDs, Faculty of Medicine, Menoufia University, Shebin Elkom, Menoufia Governorate ${ }^{3}$ Department of Dermatology and Andrology, Al Mahala General Hospital, Egyptian Health Ministry *Corresponding author: Rehab M Samaka, Mobile: +20 01002806239, Email: rehabsamaka@yahoo.com

\begin{abstract}
Background: Previous researches declared that scars are the end outcome of the natural healing and reparative process as a result of dermal fibrotic scar formation post inflammation. Few studies were conducted to explain the role of perilesional area in abnormal scar pathogenesis.

Objective: To study the histopathological changes in different types of scars and adjacent apparently non-involved skin. Hopefully, this insight can set the route for newer therapeutic approaches.

Patients and Methods: This prospective case control study was carried out on 30 participants divided into three groups. Group1 included ten keloid biopsies, Group2 included ten hypertrophic scar biopsies and Group3 included ten atrophic scar biopsies. Clinical assessment of scars was done. Skin biopsy specimens were taken from lesional and perilesional skin and sent to histopathology laboratory.

Results: Increased epidermal thickening was significantly noted in $80 \%$ of perilesional specimens of keloid scar and that was significantly higher than lesional skin, while dermal fibroblasts/myofibroblasts were mildly increased in $80 \%$ of specimens and were associated with severe perivascular inflammatory infiltrates in $70 \%$ of perilesional specimens. Myofibroblasts/ fibroblasts and perivascular infiltrate were significantly higher in perilesional than lesional keloid skin. Epidermal thickening was significantly increased in all perilesional specimens of hypertrophic scar and was significantly higher than lesional skin. In dermis, hair follicles and sebaceous glands were identified in $100 \%$ of perilesional skin. Dermal cellularity was significantly mildly increased in $100 \%$ of perilesional specimens of atrophic and hypertrophic scars and both were significantly higher than normal skin.

Conclusion: Perilesional area is a shadow area and may be a main player in abnormal scar pathogenesis and could be responsible for progression or regression of scar.
\end{abstract}

Keywords: Abnormal scars, Atrophic scar, Histopathology, Keloid, Hypertrophic scar, Perilesional area.

\section{INTRODUCTION}

Scars are the end outcome of the natural healing and reparative process as a result of dermal fibrotic scar formation post inflammation. They have been categorized according to clinical and histological appearance into various categories: keloid, hypertrophic, and atrophic scars ${ }^{(\mathbf{1})}$.

This collagen scar tissue alignment is usually of inferior functional quality to the normal collagen randomized alignment. Keloids and hypertrophic scars are caused by cutaneous injury and irritation, including trauma, insect bite, burn, surgery, vaccination, skin piercing, acne, folliculitis, chicken pox, and herpes zoster infection. Notably, superficial injuries that do reach the reticular dermis never cause keloidal and hypertrophic scarring ${ }^{(2)}$.

Keloid is a result of an overgrowth of granulation tissue at the site of a healed skin injury which is then slowly replaced by collagen type 1 . Keloids are firm, rubbery lesions or shiny, fibrous nodules, and vary from pink to red to dark brown in color sometimes accompanied by severe itchiness, pain, and changes in texture. In severe cases, it can affect movement of skin. The hypertrophic scar is defined as visible and elevated scars that do not spread into surrounding tissues and that often regress spontaneously. Unlike keloids, the hypertrophic scar reaches a certain size and subsequently stabilizes or regresses ${ }^{(3)}$.

Studies have shown that those with darker complexions are at a higher risk of keloid scarring as a result of skin trauma and less in those of a Caucasian background with no reported cases in patients with albinism. Atrophic scars are broadly described as exhibiting generalized cutaneous atrophy resulting in loss of cutaneous cells in the epidermis although appear clinically as a loss of normal dermis. Clinically, atrophic scars classically appear as depressions of the skin and commonly occur post acne amongst other causes ${ }^{(4)}$.

Structure of the skin was histologically changed in scar tissue. Collagen IV, expressed under the epidermis of normal skin, was reduced distinctly in scar tissue. Additionally, it was found that keratinocytes in scarring epidermis were more proliferative than in normal skin. These results indicate that during the skin wound healing, altered formation of basement membrane may affect the proliferation of keratinocytes, re-epithelial and tissue remodeling, and then result in scar formation (5).

Perilesional area is defined as the adjacent apparently clinically non-involved that removed to insure complete excision. The periphery is often thought 
to be responsible for the active invasive growth into the surrounding normal skin, as opposed to the less elevated central area, which shows signs of clinical regression over time. For this reason, keloid scars were divided into peripheral and central regions prior to comparison with normal skin and hypertrophic scars with respect to epidermal morphology, proliferation and differentiation (6). Clinicians have long since described the presence of an actively growing periphery as opposed to a regressive central region. However, the opposite has also been suggested, with the central area within the keloid seen as the actively growing and expanding region ${ }^{(7)}$. Few studies explored histopathological changes in perilesional tissue of abnormal scars.

Aim of this study was to investigate histopathological changes in perilesional skin, which is apparently non-involved skin, of different types of scars. This may throw light on the possible role of perilesional area in abnormal scar pathogenesis and hopefully; this insight can set the route for newer therapeutic approaches.

\section{PATIENTS AND METHODS}

This prospective case control study was performed on 30 subjects of both genders in the period from January 2018 to August 2018. These subjects were divided into 3 groups; Group1: 10 cases with keloid. Group2: 10 cases with hypertrophic scar and Group3: 10 cases with atrophic scar.

Inclusion criteria were both genders, newly diagnosed patients with keloid, hypertrophic and atrophic scars. However normal skin biopsies were collected from Plastic Surgery Department.

Exclusion criteria were the patients who had any other dermatological or systemic diseases that may affect the results. Pregnant/lactating females were also excluded.

\section{Ethical approval:}

This study was approved by Menoufia University ethics committee and was conducted in accordance with Declaration of Helsinki. A written informed consent was obtained from all patients after the procedure had been fully explained.

\section{Procedures:}

$5 \mathrm{~mm}$ punch was taken to take biopsies. Two samples were taken from every case. One sample from lesional skin and another one from perilesional skin.

Perilesional area is defined as the adjacent apparently clinically non-involved skin that removed to insure complete excision. It is $5 \mathrm{~mm}$ apart from lesion border.
All participants were subjected to detailed medical history taking including; age, gender, occupation, special habits, onset, course, duration of the disease and site of lesions with clinical assessment of scars.

Skin biopsy specimens were taken from patients and controls and preserved in $10 \%$ formalin then sent to Pathology Department, Faculty of Medicine Menoufia University for routine processing and cutting.

\section{Histopathological changes:}

- Epidermis and dermis were assessed.

- Epidermis for: epidermal thickness, epidermal rete ridges.

- Dermis for: cellularity, vascularity, hair follicles, sebaceous glands, fibroblast/myofibroblasts, collagen fiber density, collagen fiber orientation, collagen fiber size, collagen fiber status, collagen fiber arrangement, collagen fiber pattern, collagen fiber direction, dermal inflammatory infiltrates and degree of inflammatory infiltrates ${ }^{(8)}$.

- Assessment of hematoxylin and eosin (H and $\mathrm{E})$ stained slides for different histopathological changes in epidermis and dermis.

\section{Statistical analysis}

Data were collected, tabulated and statistically analyzed using a personal computer with "Statistical Package for the Social Sciences (SPSS), version 22, Armonk, NY: IBM Corp. Data are presented as frequency and percentage. Contingency tables were analyzed with the following tests: Chi- square test $\left(\mathrm{X}^{2}\right.$ test) and Fisher's exacts test. Differences were considered significant $(\mathrm{S})$ when $(\mathrm{P}<0.05)$.

\section{RESULTS}

On comparison between lesional and perilesional keloid regarding epidermal and dermal changes there were increased epidermal thickening and normal rete ridges in $80 \%$ of perilesional specimens. Regarding dermal changes, all perilesional specimens had normal vascularity with increased cellularity.

Hair follicles were present in 50\% of perilesional specimens and sebaceous glands were present in all of perilesional specimens. Fibroblast/myofibroblasts were increased in $80 \%$ of perilesional specimens. Collagen fiber density and orientation were normal in all perilesional specimens. The arrangement of collagen fibers in all perilesional specimens were loose. The degree of inflammatory infiltrates was marked in $70 \%$ of perilesional specimens (Table 1 and Figure 1). 
Table (1): Comparison between lesional and perilesional keloid regarding epidermal and dermal changes

\begin{tabular}{|c|c|c|c|c|c|}
\hline & Parameters & \begin{tabular}{|c|} 
Lesional keloid \\
$(\mathrm{No}=10)$ \\
No $(\%)$ \\
\end{tabular} & \begin{tabular}{|c|} 
Perilesional keloid \\
$($ No $=10)$ \\
No $(\%)$ \\
\end{tabular} & $\begin{array}{c}\text { Test of } \\
\text { significance }\end{array}$ & $P$ value \\
\hline Epidermal thickness & $\begin{array}{l}\text { Decreased } \\
\text { Increased }\end{array}$ & $\begin{array}{c}10(100) \\
0(0)\end{array}$ & \begin{tabular}{|l}
$2(20)$ \\
$8(80)$
\end{tabular} & $\mathrm{FE}=\mathbf{1 3 . 3 3}$ & $0.001 *$ \\
\hline Epidermal rete ridges & $\begin{array}{c}\text { Lacking } \\
\text { Partial } \\
\text { Normal } \\
\end{array}$ & $\begin{array}{c}6(60) \\
4(40) \\
0(0)\end{array}$ & $\begin{array}{l}1(10) \\
1(10) \\
8(80) \\
\end{array}$ & $\mathbf{F E}=\mathbf{1 3 . 3 7}$ & $0.001 *$ \\
\hline Dermal cellularity & $\begin{array}{c}\text { Mild increase } \\
\text { Moderate increase } \\
\text { Marked increase }\end{array}$ & $\begin{array}{c}1(10) \\
0(0) \\
9(90) \\
\end{array}$ & $\begin{array}{c}0(0) \\
10(100) \\
0(0)\end{array}$ & $\mathbf{F E}=20$ & $<0.001 *$ \\
\hline Dermal vascularity & $\begin{array}{c}\text { Decrease } \\
\text { Normal }\end{array}$ & $\begin{array}{c}10(100) \\
0(0)\end{array}$ & $\begin{array}{c}0(0) \\
10(100)\end{array}$ & $F E=20$ & $<0.001 *$ \\
\hline Hair follicle & $\begin{array}{l}\text { Lacking } \\
\text { Present }\end{array}$ & $\begin{array}{l}9(90) \\
1(10)\end{array}$ & $\begin{array}{l}5(50) \\
5(50)\end{array}$ & $\mathbf{X}^{2}=3.81$ & $<0.051$ \\
\hline Sebaceous gland & $\begin{array}{l}\text { Lacking } \\
\text { Present }\end{array}$ & $\begin{array}{c}10(100) \\
0(0)\end{array}$ & $\begin{array}{c}0(0) \\
10(100)\end{array}$ & $\mathbf{F E}=\mathbf{2 0}$ & $<0.001 *$ \\
\hline Fibroblast/myofibroblasts & $\begin{array}{c}\text { Mild } \\
\text { Moderate } \\
\text { Marked }\end{array}$ & $\begin{array}{c}0(0) \\
0(0) \\
10(100)\end{array}$ & $\begin{array}{c}8(80) \\
2(20) \\
0(0)\end{array}$ & $\mathrm{FE}=20$ & $<0.001 *$ \\
\hline Collagen fiber density & $\begin{array}{c}\text { Abnormal } \\
\text { Normal }\end{array}$ & $\begin{array}{c}10(100) \\
0(0)\end{array}$ & $\begin{array}{c}0(0) \\
10(100)\end{array}$ & $\mathbf{F E}=\mathbf{2 0}$ & $<0.001 *$ \\
\hline Collagen fiber orientation & $\begin{array}{c}\text { Abnormal } \\
\text { Normal }\end{array}$ & $\begin{array}{c}10(100) \\
0(0)\end{array}$ & $\begin{array}{c}0(0) \\
10(100)\end{array}$ & $\mathrm{FE}=\mathbf{2 0}$ & $<0.001^{*}$ \\
\hline Collagen fiber size & $\begin{array}{l}\text { Small } \\
\text { Large } \\
\text { Mixed }\end{array}$ & $\begin{array}{l}2(20) \\
2(20) \\
6(60)\end{array}$ & $\begin{array}{c}4(40) \\
0(0) \\
6(60)\end{array}$ & $\mathrm{FE}=2.67$ & 0.26 \\
\hline $\begin{array}{l}\text { Collagen bundle } \\
\text { status }\end{array}$ & $\begin{array}{l}\text { Thin } \\
\text { Thick }\end{array}$ & $\begin{array}{c}10(100) \\
0(0)\end{array}$ & $\begin{array}{c}0(0) \\
10(100)\end{array}$ & $\mathbf{F E}=\mathbf{2 0}$ & $<0.001 *$ \\
\hline $\begin{array}{l}\text { Collagen fiber } \\
\text { arrangement }\end{array}$ & $\begin{array}{l}\text { Closed } \\
\text { Loose }\end{array}$ & $\begin{array}{l}5(50) \\
5(50)\end{array}$ & $\begin{array}{c}0(0) \\
10(100)\end{array}$ & $\mathrm{FE}=6.67$ & $0.01 *$ \\
\hline Collagen fiber direction & $\begin{array}{l}\text { Parallel } \\
\text { Mixed }\end{array}$ & $\begin{array}{l}1(10) \\
9(90)\end{array}$ & $\begin{array}{c}0(0) \\
10(100)\end{array}$ & $\mathrm{FE}=1.05$ & 0.305 \\
\hline $\begin{array}{c}\text { Dermal inflammatory } \\
\text { infiltrate }\end{array}$ & $\begin{array}{l}\text { Present } \\
\text { Absent }\end{array}$ & $\begin{array}{l}9(90) \\
1(10)\end{array}$ & $\begin{array}{c}10(100) \\
0(0)\end{array}$ & $\mathrm{FE}=1.05$ & 0.305 \\
\hline $\begin{array}{c}\text { Degree of inflammatory } \\
\text { infiltrate }\end{array}$ & $\begin{array}{c}\text { Mild } \\
\text { Moderate } \\
\text { Marked }\end{array}$ & $\begin{array}{c}10(100) \\
0(0) \\
0(0)\end{array}$ & $\begin{array}{l}0(0) \\
3(30) \\
7(70)\end{array}$ & $\mathrm{FE}=\mathbf{2 0 . 0}$ & $<0.001 *$ \\
\hline
\end{tabular}

N.B: Collagen fiber pattern was haphazardly arranged in all lesional and perilesional groups. *: Significant;

$\mathrm{X}^{2}$ : Chi square test, FE: Fisher's Exact test

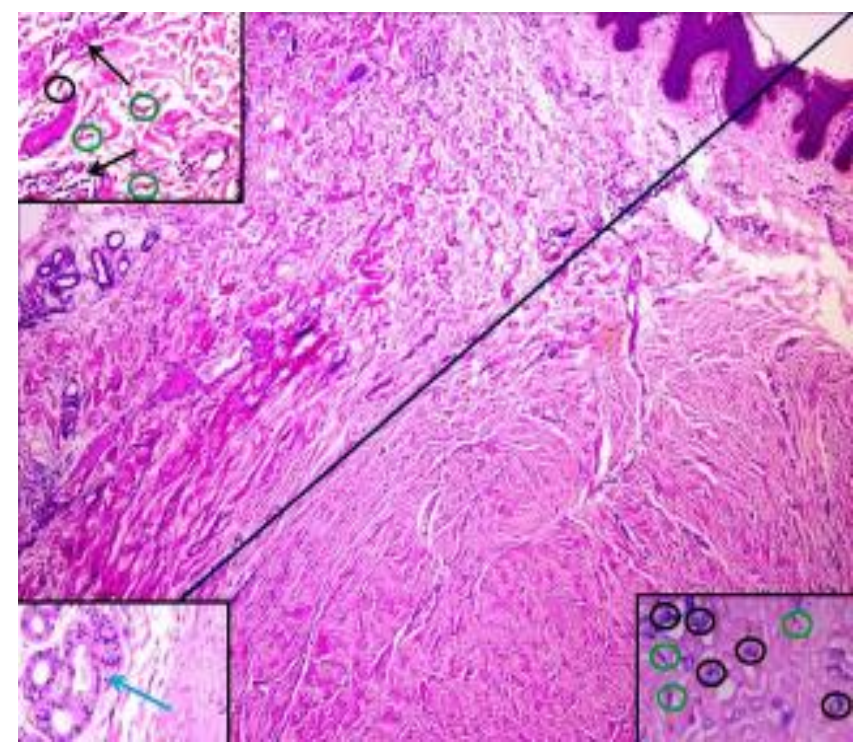

Figure (1): Keloid case showed in right lower half, the lesional area with busy dermis occupied by dense thick collagen bundles (inset) high power view of cellular components, fibroblasts (green circles) and myofibroblasts. There were significant differences between perilesional and lesional areas of hypertrophic scar regarding to epidermal and dermal changes. Epidermal changes showed significant increase in epidermal thickness in all perilesional specimens with normal rete ridges in $60 \%$. Regarding dermal changes, cellularity was mildly increased in $80 \%$ of perilesional specimens. In all perilesional specimens, vascularity was normal, fibroblasts/myofibroblasts were mildly increased, collagen fibers were thin with even distributed pattern and its direction was parallel to epidermis. Hair collagen fiber density and orientation were normal and follicles and sebaceous glands were identified in all of perilesional biopsies. All perilesional specimens had small size of collagen fibers and their arrangement were loose (Table 2 and Figure 2). 
`https://ejhm.journals.ekb.eg/

Table (2): Comparison between lesional and perilesional hypertrophic regarding epidermal and dermal changes

\begin{tabular}{|c|c|c|c|c|c|c|}
\hline & \multicolumn{2}{|l|}{ Parameters } & \begin{tabular}{|c} 
Lesional \\
hypertrophic \\
scar \\
$(\mathrm{No}=10)$
\end{tabular} & $\begin{array}{c}\text { Perilesional } \\
\text { Hypertrophic } \\
\text { scar }(\text { No = 10) }\end{array}$ & $\begin{array}{c}\text { Test of } \\
\text { significance }\end{array}$ & $P$ value \\
\hline Epidermal thickness & $\begin{array}{c}\text { Decreased } \\
\text { Increased } \\
\text { Normal }\end{array}$ & $\begin{array}{l}\text { No } \\
(\%)\end{array}$ & $\begin{array}{c}10(100) \\
0(0) \\
0(0)\end{array}$ & $\begin{array}{c}0(0) \\
10(100) \\
0(0)\end{array}$ & $\mathrm{FE}=20$ & $<0.001 *$ \\
\hline Epidermal rete ridges & $\begin{array}{c}\text { Lacking } \\
\text { Partial } \\
\text { Normal }\end{array}$ & $\begin{array}{l}\text { No } \\
(\%)\end{array}$ & $\begin{array}{c}7(70) \\
3(30) \\
0(0)\end{array}$ & $\begin{array}{l}3(30) \\
1(10) \\
6(60)\end{array}$ & $\mathrm{FE}=11.4$ & $0.01 *$ \\
\hline Dermal cellularity & \begin{tabular}{|c|} 
Mild increase \\
Moderate increase \\
Marked increase
\end{tabular} & $\begin{array}{l}\text { No } \\
(\%)\end{array}$ & $\begin{array}{c}10(100) \\
0(0) \\
0(0)\end{array}$ & $\begin{array}{c}0(0) \\
8(80) \\
2(20)\end{array}$ & $\mathrm{FE}=20$ & $<0.001 *$ \\
\hline Dermal vascularity & $\begin{array}{c}\text { Decrease } \\
\text { Normal }\end{array}$ & $\begin{array}{l}\text { No } \\
(\%)\end{array}$ & $\begin{array}{c}10(10) \\
0(0)\end{array}$ & $\begin{array}{c}0(0) \\
10(100)\end{array}$ & $\mathrm{FE}=20$ & $<0.001 *$ \\
\hline Hair follicle & $\begin{array}{l}\text { Lacking } \\
\text { Present }\end{array}$ & $\begin{array}{l}\text { No } \\
(\%)\end{array}$ & $\begin{array}{c}10(100) \\
0(0)\end{array}$ & $\begin{array}{c}0(0) \\
10(100)\end{array}$ & $\mathrm{FE}=20$ & $<0.001 *$ \\
\hline Sebaceous gland & $\begin{array}{r}\text { Lacking } \\
\text { Present }\end{array}$ & $\begin{array}{l}\text { No } \\
(\%)\end{array}$ & $\begin{array}{c}10(100) \\
0\end{array}$ & $\begin{array}{c}0(0) \\
10(100)\end{array}$ & $\mathrm{FE}=20$ & $<0.001 *$ \\
\hline Fibroblast/myofibroblasts & $\begin{array}{c}\text { Mild } \\
\text { Moderate } \\
\text { Marked }\end{array}$ & $\begin{array}{l}\text { No } \\
(\%)\end{array}$ & $\begin{array}{c}0(0) \\
10(100) \\
0(0)\end{array}$ & $\begin{array}{c}10(100) \\
0(0) \\
0(0)\end{array}$ & $\mathrm{FE}=20$ & $<0.001 *$ \\
\hline Collagen fiber density & $\begin{array}{c}\text { Abnormal } \\
\text { Normal }\end{array}$ & $\begin{array}{l}\text { No } \\
(\%)\end{array}$ & $\begin{array}{c}10(100) \\
0(0)\end{array}$ & $\begin{array}{c}0(0) \\
10(100)\end{array}$ & $\mathrm{FE}=20$ & $<0.001 *$ \\
\hline Collagen fiber orientation & $\begin{array}{c}\text { Abnormal } \\
\text { Normal }\end{array}$ & $\begin{array}{l}\text { No } \\
(\%)\end{array}$ & $\begin{array}{c}10(100) \\
0(0)\end{array}$ & $\begin{array}{c}0(0) \\
10(100)\end{array}$ & $\mathrm{FE}=20$ & $<0.001 *$ \\
\hline Collagen fiber size & $\begin{array}{c}\text { Small } \\
\text { Large } \\
\text { Mixed } \\
\end{array}$ & $\begin{array}{l}\text { No } \\
(\%)\end{array}$ & $\begin{array}{c}3(30) \\
7(70) \\
0(0)\end{array}$ & $\begin{array}{c}10(100) \\
0(0) \\
0(0)\end{array}$ & $\mathrm{FE}=10.77$ & $0.001 *$ \\
\hline Collagen fiber status & $\begin{array}{l}\text { Thin } \\
\text { Thick }\end{array}$ & $\begin{array}{l}\text { No } \\
(\%)\end{array}$ & $\begin{array}{c}0(0) \\
10(100)\end{array}$ & $\begin{array}{c}10(100) \\
0(0)\end{array}$ & $\mathrm{FE}=20$ & $<0.001 *$ \\
\hline $\begin{array}{c}\text { Collagen fiber } \\
\text { arrangement }\end{array}$ & $\begin{array}{l}\text { Closed } \\
\text { Loose }\end{array}$ & $\begin{array}{l}\text { No } \\
(\%)\end{array}$ & $\begin{array}{l}5(50) \\
5(50)\end{array}$ & $\begin{array}{c}0(0) \\
10(100)\end{array}$ & $\mathrm{FE}=6.67$ & $0.03^{*}$ \\
\hline Collagen fiber pattern & $\begin{array}{l}\text { Haphazard } \\
\text { Even } \\
\text { Mixed }\end{array}$ & $\begin{array}{l}\text { No } \\
(\%)\end{array}$ & $\begin{array}{c}10(100) \\
0(0) \\
0(0)\end{array}$ & $\begin{array}{c}0(0) \\
10(100) \\
0(0)\end{array}$ & $\mathrm{FE}=\mathbf{2 0}$ & $<0.001 *$ \\
\hline $\begin{array}{c}\text { Collagen fiber direction } \\
\text { pattern }\end{array}$ & $\begin{array}{c}\text { Parallel } \\
\text { Perpendicular } \\
\text { Mixed pattern }\end{array}$ & $\begin{array}{l}\text { No } \\
(\%)\end{array}$ & $\begin{array}{c}0(0) \\
0(0) \\
10(100)\end{array}$ & $\begin{array}{c}10(100) \\
0(0) \\
0(0)\end{array}$ & $\mathrm{FE}=\mathbf{2 0}$ & $<0.001 *$ \\
\hline $\begin{array}{c}\text { Dermal inflammatory } \\
\text { infiltrate }\end{array}$ & $\begin{array}{c}\text { Absent } \\
\text { Preset }\end{array}$ & $\begin{array}{l}\text { No } \\
(\%)\end{array}$ & $\begin{array}{l}1(10) \\
9(90) \\
\end{array}$ & $\begin{array}{c}0(0) \\
10(100)\end{array}$ & $\mathrm{FE}=1.05$ & 0.305 \\
\hline $\begin{array}{c}\text { Degree of inflammatory } \\
\text { infiltrate }\end{array}$ & $\begin{array}{c}\text { Mild } \\
\text { Moderate } \\
\text { Marked }\end{array}$ & $\begin{array}{l}\text { No } \\
(\%)\end{array}$ & $\begin{array}{c}10(100) \\
0(0) \\
0(0)\end{array}$ & $\begin{array}{c}6(60) \\
4(40) \\
0(0)\end{array}$ & $\mathrm{FE}=\mathbf{5}$ & $0.025^{*}$ \\
\hline
\end{tabular}

*: Significant; $\mathrm{X}^{2}$ : Chi square test, FE: Fisher's Exact test. 


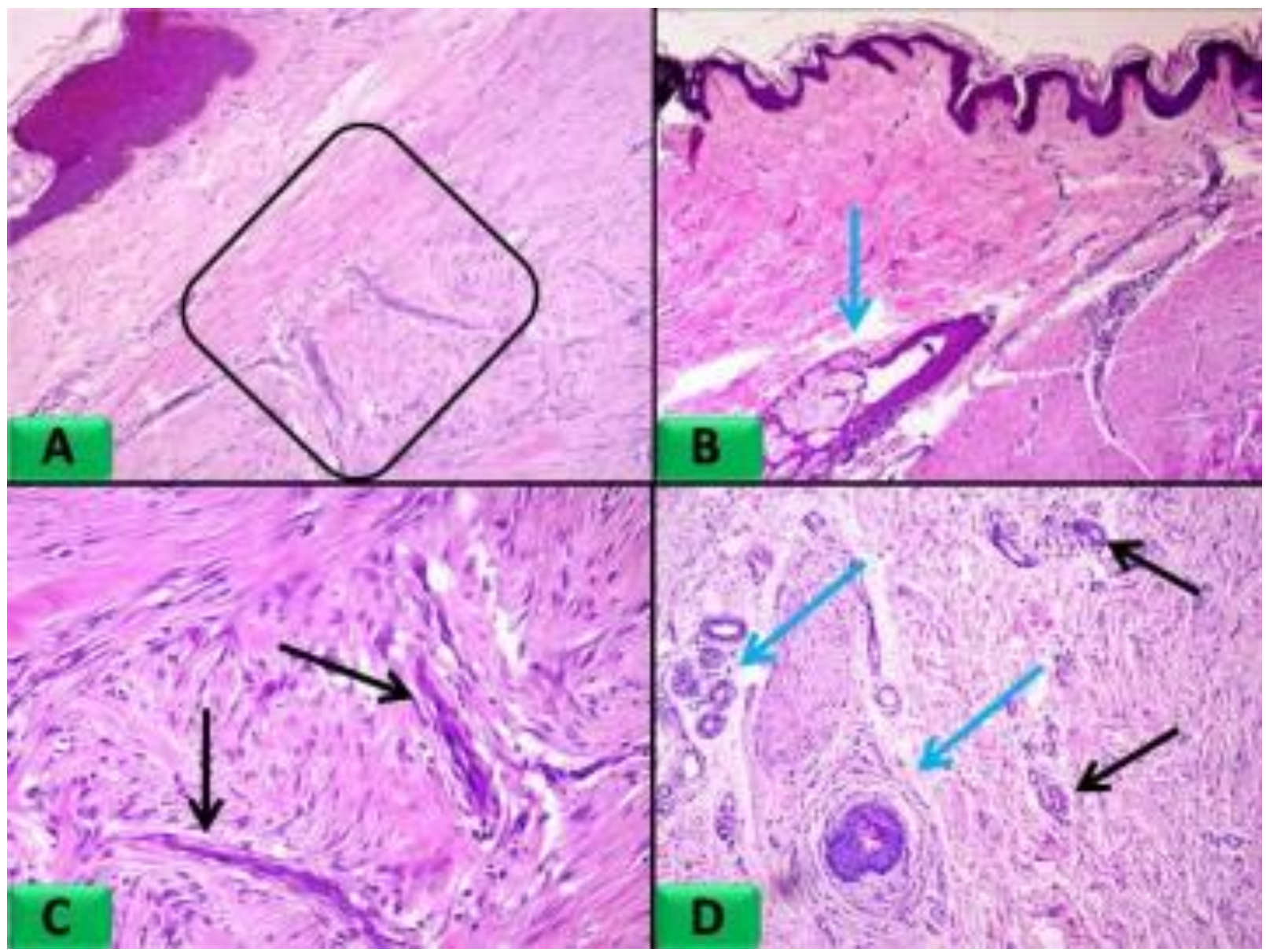

Figure (2): A) Hypertrophic scar showed perpendicular blood vessels to surface epithelium and parallel fibroblasts/myofibroblasts (square). B) Perilesional area showed epidermis with unremarkable pathological changes. C) High power view of figure (A), demonstrating blood vessels (arrows) and fibroblasts/myofibroblasts between them. D) Dermis of perilesional skin demonstrates preserved adnexa (blue arrows) and mild congested blood vessels (black arrows).

Also there were significant differences between perilesional and lesional epidermal and dermal changes in atrophic scar. There was normal epidermal thickness in $80 \%$ of perilesional specimens. All perilesional specimens had normal rete ridges. Regarding dermal changes, all perilesional specimens had mildly increased cellularity, hair follicles and sebaceous glands were present, fibroblast/myofibroblasts were mildly increased, collagen fiber density and orientation were normal. Vascularity was normal in all of perilesional sections. All perilesional specimens had small size of collagen fibers and their arrangement was loose. All perilesional specimens had thin collagen fibers (Table 3 and Figure 3). 
`https://ejhm.journals.ekb.eg/

Table (3): Comparison between lesional and perilesional atrophic regarding epidermal and dermal changes

\begin{tabular}{|c|c|c|c|c|}
\hline Parameters & \begin{tabular}{|c|} 
Lesional \\
trophic scar \\
No $=10$ \\
No $(\%)$ \\
\end{tabular} & $\begin{array}{c}\text { Perilesional } \\
\text { atrophic scar } \\
\text { No }=10 \\
\text { No }(\%)\end{array}$ & est of significance & $P$ value \\
\hline $\begin{array}{l}\text { Epidermal thickness } \\
\text { Decreased } \\
\text { Increased } \\
\text { Normal }\end{array}$ & $\begin{array}{l}9(90) \\
1(10) \\
0(0)\end{array}$ & $\begin{array}{l}2(20) \\
0(0) \\
8(80)\end{array}$ & $\mathrm{FE}=13.46$ & $0.001 *$ \\
\hline $\begin{array}{c}\text { Epidermal rete ridges } \\
\text { Lacking } \\
\text { Partial } \\
\text { Normal } \\
\end{array}$ & $\begin{array}{l}5(50) \\
5(50) \\
0(0) \\
\end{array}$ & $\begin{array}{c}0(0) \\
0(0) \\
10(100) \\
\end{array}$ & $\mathrm{FE}=20$ & $<0.001 *$ \\
\hline $\begin{array}{l}\text { Dermal cellularity } \\
\text { Increased } \\
\text { Mild increase } \\
\text { Moderate increase } \\
\text { Marked increase }\end{array}$ & $\begin{array}{l}9(90) \\
0(0) \\
0(0) \\
1(10)\end{array}$ & $\begin{array}{c}0(0) \\
10(100) \\
0(0) \\
0(0)\end{array}$ & $\mathrm{FE}=20$ & $<0.001 *$ \\
\hline $\begin{array}{l}\text { Dermal vascularity } \\
\text { Increase } \\
\text { Normal }\end{array}$ & $\begin{array}{l}5(50) \\
5(50)\end{array}$ & $\begin{array}{c}0(0) \\
10(100)\end{array}$ & $\mathrm{FE}=6.67$ & $0.03 *$ \\
\hline $\begin{array}{c}\text { Hair follicle } \\
\text { Lacking } \\
\text { Present }\end{array}$ & $\begin{array}{c}10(100) \\
0(0)\end{array}$ & $\begin{array}{c}0(0) \\
10(100)\end{array}$ & $\mathrm{FE}=20$ & $0<0.001 *$ \\
\hline $\begin{array}{c}\text { Sebaceous gland } \\
\text { Lacking } \\
\text { Present }\end{array}$ & $\begin{array}{c}10(100) \\
0(0)\end{array}$ & $\begin{array}{c}0(0) \\
10(100)\end{array}$ & $\mathrm{FE}=20$ & $<0.001 *$ \\
\hline $\begin{array}{c}\text { Fibroblast/myofibroblasts } \\
\text { Mild } \\
\text { Moderate } \\
\text { Marked } \\
\end{array}$ & $\begin{array}{l}1(10) \\
2(20) \\
7(70)\end{array}$ & $\begin{array}{c}10(100) \\
0(0) \\
0(0)\end{array}$ & $\mathrm{FE}=16.36$ & $<0.001 *$ \\
\hline $\begin{array}{c}\text { Collagen fiber density } \\
\text { Abnormal } \\
\text { Normal } \\
\end{array}$ & $\begin{array}{c}10(100) \\
0(0)\end{array}$ & $\begin{array}{c}0(0) \\
10(100)\end{array}$ & $\mathrm{FE}=20$ & $0<0.001 *$ \\
\hline $\begin{array}{c}\text { Collagen fiber orientation } \\
\text { Abnormal } \\
\text { Normal } \\
\end{array}$ & $\begin{array}{c}10(100) \\
0(0)\end{array}$ & $\begin{array}{c}0(0) \\
10(100)\end{array}$ & $\mathrm{FE}=20$ & $<0.001 *$ \\
\hline $\begin{array}{c}\text { Collagen fiber size } \\
\text { Small } \\
\text { Large } \\
\text { Mixed } \\
\end{array}$ & $\begin{array}{c}0(0) \\
0(0) \\
10(100)\end{array}$ & $\begin{array}{c}10(100) \\
0(0) \\
0(0)\end{array}$ & $\mathrm{FE}=20$ & $<0.001 *$ \\
\hline $\begin{array}{c}\text { Collagen fiber status } \\
\text { Thin } \\
\text { Thick } \\
\text { Mixed } \\
\end{array}$ & $\begin{array}{c}0(0) \\
0(0) \\
10(100) \\
\end{array}$ & $\begin{array}{c}10(100) \\
0(0) \\
0(0) \\
\end{array}$ & $\mathrm{FE}=20$ & $<0.001 *$ \\
\hline $\begin{array}{c}\text { Collagen fiber arrangement } \\
\text { Closed } \\
\text { Loose } \\
\text { Mixed }\end{array}$ & $\begin{array}{l}4(40) \\
3(30) \\
3(30)\end{array}$ & $\begin{array}{c}0(0) \\
10(100) \\
0(0)\end{array}$ & $\mathrm{FE}=10.77$ & $0.005^{*}$ \\
\hline $\begin{array}{c}\text { egree of inflammatory infiltrate } \\
\text { Mild } \\
\text { Moderate } \\
\text { Marked } \\
\end{array}$ & $\begin{array}{c}10(100) \\
0(0) \\
0(0)\end{array}$ & $\begin{array}{l}8(80) \\
2(20) \\
0(0)\end{array}$ & $\mathrm{FE}=2.22$ & 0.136 \\
\hline
\end{tabular}


N.B: In all cases of lesional and perilesional groups there were haphazard collagen fiber pattern, parallel collagen fiber direction and presence of dermal inflammatory infiltrate.

*: Significant; $\mathrm{X}^{2}$ : Chi square test; FE: Fisher's Exact test.

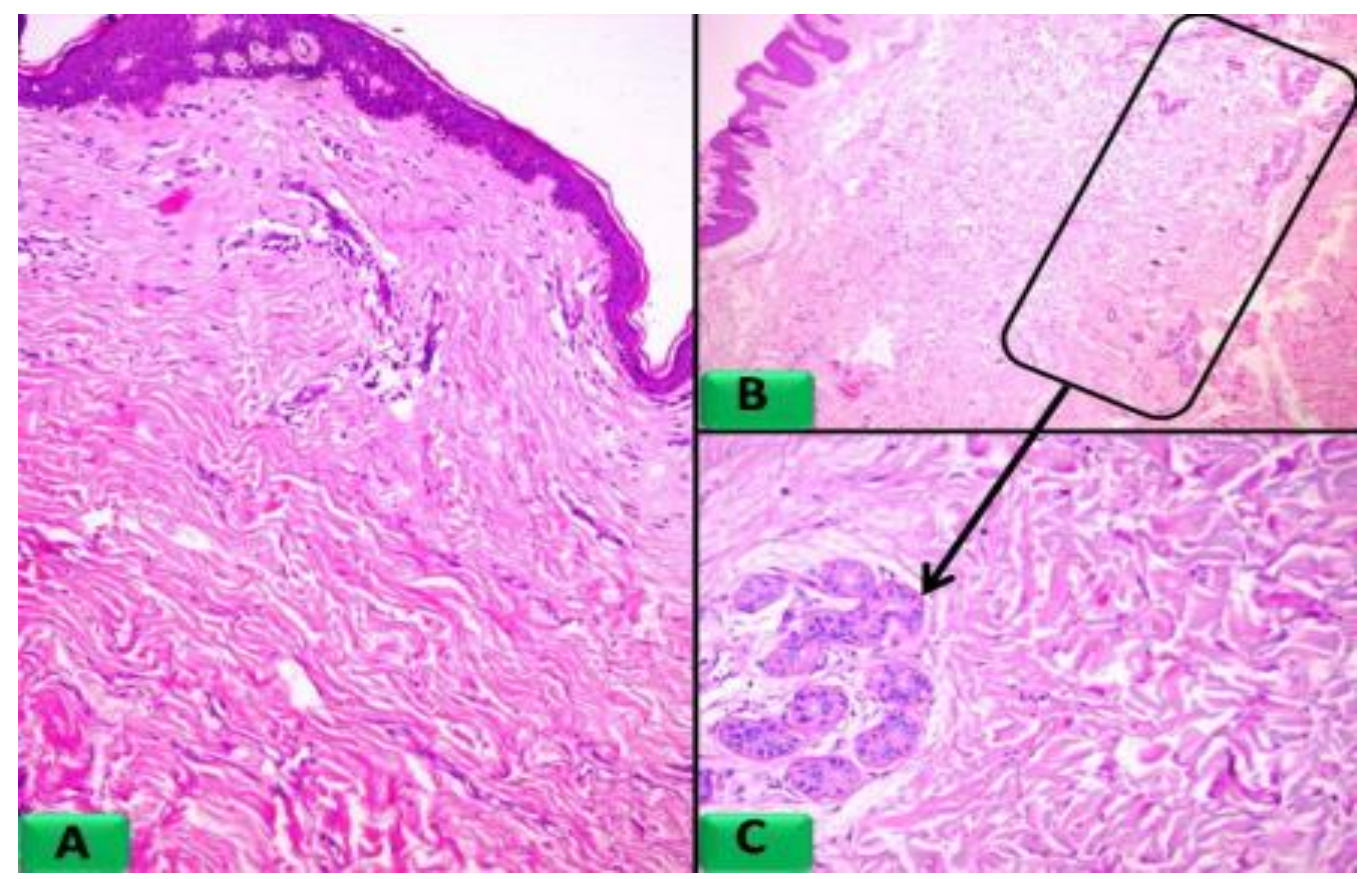

Figure (3): A) Atrophic scar showed atrophy of epidermis and loss of rete ridges B) Perilesional area showed epidermis with unremarkable pathological changes Dermis showed preserved eccrine sweat gland (rectangle). C) High power view of (B) demonstrating ducts of eccrine sweat glands ( $\mathrm{H}$ and $\mathrm{E} A$ and $\mathrm{C} \times 100$ and $\mathrm{B} \times 40$ ).

\section{DISCUSSION}

Scars are the end outcome of the natural healing and reparative process as a result of dermal fibrotic scar formation post inflammation. They were categorized according to clinical and histological appearance into various categories: keloid, hypertrophic, and atrophic scars Patel et al. ${ }^{(1)}$.

In this study, there was a significant difference between perilesional and lesional keloid regarding to epidermal thickening and epidermal rete ridges as most of perilesional specimens had increased epidermal thickening and had normal rete ridges. This was in accordance with Limandjaja $\boldsymbol{e t}$ al. ${ }^{\left({ }^{(9)}\right.}$ in their study, who reported that keloids showed increased epidermal thickness compared with normal skin and hypertrophic scars. Their findings indicate that the epidermis is associated with keloid pathogenesis. This was not due to hyperproliferation, but possibly caused by abnormal early terminal differentiation, which affects stratum corneum formation ${ }^{(\mathbf{1 0})}$. Therefore, it is possible that keratinocytes of perilesional area also participate in abnormal wound healing processes leading to formation of keloid scars.

In the current study, fibroblast/myofibroblasts were significantly increased in most of perilesional keloid specimens. All perilesional specimens had both small and large collagen fibers and their arrangement was loose. Collagen fiber density and orientation were normal and the degree of inflammatory infiltrates was marked. This was opposite to Syed et al. ${ }^{(11)}$ who demonstrated that biopsies taken from the growing margin (perilesional) of keloid scars show a greater collagen production than those taken from different sites from the same keloid lesion.

However, this was nearly matched with Ashcroft et $\boldsymbol{a l} .{ }^{\text {(7) }}$ in their study who demonstrated that perilesional and intra-lesional keloid fibroblast conditioned media induce significantly elevated metabolic viability and proliferation in normal scar and skin fibroblasts, So perilesional area of keloid may be responsible for its progression and growth beyond the boundaries of the original wound.

During the proliferative phase of healing, macrophages in the area of injury release growth factors, such as transforming growth factor $\beta$ (TGF- $\beta$ ), which activates fibroblasts to create collagen. In hypertrophic scars and keloids, the thinking is that the dysregulation of TGF- $\beta$ leads to the formation of these types of scars. TGF- $\beta 1$ and 2 manage activation of fibroblasts. The belief is that overexpression of TGF- $\beta$ 1 and 2 and decreased expression of TGF- $\beta 3$ is what leads to increased extracellular matrix production causing these abnormal scars ${ }^{\mathbf{( 1 2})}$.

In this study, significant increase in epidermal thickness with normal rete ridges was noted in all perilesional specimens of hypertrophic scars. This was nearly matched with Hakvoort et $\boldsymbol{a l} .{ }^{(13)}$ who reported that hypertrophic scars showed increased proliferation and increased epidermal thickness. This is due to increased expression of the keratinocyte 
hyperproliferation and activation markers cytokines 6 , 16 and 17. These early epidermal abnormalities do suggest that the epidermal compartment is involved in the pathogenesis of this abnormal scar ${ }^{(14)}$.

In current study there were significant differences between lesional and perilesional hypertrophic scar tissue regarding to collagen fiber size, collagen fiber arrangement and collagen fiber direction similar to keloid lesions described above. This was in accordance with Huang $\boldsymbol{e t} \boldsymbol{a l} .{ }^{(15)}$ who reported that keloids and hypertrophic scars to be distinct types of scar and there were a significant differences between perilesional and lesional hypertrophic regarding to collagen fiber density, collagen fiber status and collagen fiber arrangement. Keloid has thick eosinophilic (hyalinizing) collagen bundles called "keloidal collagen": these are present in keloids but fewer in hypertrophic scars. Thus, it is possible that hypertrophic scars and keloids are actually manifestations of the same fibroproliferative skin disorder and they just differ in the intensity and duration of inflammation ${ }^{(2)}$.

In this study there were significant differences between lesional and perilesional atrophic scar tissue regarding to epidermal changes as most of perilesional sections had normal epidermal thickness and normal rete ridges. This was nearly matched with Butler $\boldsymbol{e t}$ al. (16) who explained these findings by degradation of elastic fibers and collagen fibers occurred in the dermis of atrophic lesion, followed by their incomplete recovery. Abnormally excessive inflammation mediated by innate immunity with $\mathrm{T}$ helper 17 and $\mathrm{T}$ helper 1 cells was observed. Epidermal proliferation was significantly diminished. Transforming growth factor (TGF- $\beta 1$ ) was drastically elevated in patients who were prone to scar (APS), suggesting that aberrant TGF- $\beta 1$ signaling is an underlying modulator of all of these pathological processes ${ }^{(17)}$.

\section{CONCLUSION}

Histopathological changes of perilesional areas in different types of scar throw the light on dynamic processes. These areas could be responsible for progression or regression of scar. So perilesional area is a shadow area and may be the main player in pathogenesis of scar and this could open the gate for new target therapy for this disease. Further studies are required to establish these findings.

\section{REFERENCES}

1. Patel L, McGrouther D, Chakrabarty K (2014): Evaluating evidence for atrophic scarring treatment modalities. JRSM Open, 5(9):2054-9.

2. Ogawa R (2017): Keloid and hypertrophic scars are the result of chronic inflammation in the reticular dermis.
International Journal of Molecular Sciences, 18(3):6068.

3. Rabello F, Souza C, Farina J (2014): Update on hypertrophic scar treatment. Clinics, 69:565-73.

4. Andrews J, Marttala J, Macarak E et al. (2016): Keloids: The paradigm of skin fibrosisPathomechanisms and treatment. Matrix Biology, 51:3746.

5. Yang S, Geng Z, Ma K et al. (2016): Comparison of the histological morphology between normal skin and scar tissue. Journal of Huazhong University of Science and Technology, 36(2):265-9.

6. Lu F, Gao J, Ogawa R et al. (2007): Biological differences between fibroblasts derived from peripheral and central areas of keloid tissues. Plastic and Reconstructive Surgery, 120(3):625-30.

7. Ashcroft K, Syed F, Bayat A (2013): Site-specific keloid fibroblasts alter the behaviour of normal skin and normal scar fibroblasts through paracrine signalling. PloS One, 8(12):75800-5.

8. Vercelli S, Ferriero G, Sartorio F et al. (2003): How to assess postsurgical scars: a review of outcome measures. Disability and Rehabilitation, 25(31):2055-63.

9. Limandjaja G, van den Broek L, Waaijman T et al. (2017): Increased epidermal thickness and abnormal epidermal differentiation in keloid scars. British Journal of Dermatology, 176(1):116-26.

10. Gauglitz G, Korting H, Pavicic T et al. (2011): Hypertrophic scarring and keloids: pathomechanisms and current and emerging treatment strategies. Molecular Medicine, 17(1-2):113-25.

11. Syed F, Ahmadi E, Iqbal S et al. (2011): Fibroblasts from the growing margin of keloid scars produce higher levels of collagen I and III compared with intralesional and extralesional sites: clinical implications for lesional site-directed therapy. British Journal of Dermatology, 164(1):83-96.

12. Berman B, Maderal A, Raphael B (2017): Keloids and hypertrophic scars: pathophysiology, classification, and treatment. Dermatologic Surgery, 43:3-18.

13. Hakvoort T, Altun V, Ramrattan $R$ et al. (1999): Epidermal participation in post-burn hypertrophic scar development. Virchows Archiv., 434(3):221-6.

14. Andriessen $M$, Niessen $F$, van de Kerkhof $P$ et al. (1998): Hypertrophic scarring is associated with epidermal abnormalities: an immunohistochemical study. The Journal of Pathology: A Journal of the Pathological Society of Great Britain and Ireland, 186(2):192-200.

15. Huang C, Akaishi S, Hyakusoku H et al. (2014): Are keloid and hypertrophic scar different forms of the same disorder? A fibroproliferative skin disorder hypothesis based on keloid findings. International Wound Journal, 11(5):517-22.

16. Butler $P$, Longaker $M$, Yang $G$ (2008): Current progress in keloid research and treatment. Journal of the American College of Surgeons, 206(4):731-41.

17. Moon J, Yoon J, Yang J et al. (2019): Atrophic acne scar: a process from altered metabolism of elastic fibres and collagen fibres based on transforming growth factor$\beta 1$ signalling. British Journal of Dermatology, 181(6):1226-37. 